\title{
Erratum to: Some Higher Order Newton-Like Methods for Solving System of Nonlinear Equations and Its Applications
}

\section{Kalyanasundaram Madhu' ${ }^{1}$. Jayakumar Jayaraman ${ }^{1}$ (D)}

\section{Erratum to: Int. J. Appl. Comput. Math (2017) 3:2213-2230 DOI 10.1007/s40819-016-0234-z}

The authors regret the fact that throughout the section of Efficiency of the Methods, due to MATLAB code typos, the following errors were found in Table 1 and Fig. 1. For example, we have used 2nd NM, EI is $\frac{2^{1}}{n+n^{2}}$ instead of $2 \frac{1}{n+n^{2}}$ and CE is $\frac{2^{1}}{\frac{1}{3} n^{3}+2 n^{2}+\frac{2}{3} n}$ instead of $2 \frac{1}{\frac{1}{3} n^{3}+2 n^{2}+\frac{2}{3} n}$. Similarly we have used all the methods.

The authors would like to apologise for any inconvenience caused.

It was displayed as follows:

The online version of the original article can be found under doi:10.1007/s40819-016-0234-z.

Jayakumar Jayaraman jjayakumar@pec.edu

Kalyanasundaram Madhu kalyan742@pec.edu

1 Department of Mathematics, Pondicherry Engineering College, Pondicherry 605014, India 
Table 1 Comparison of EI and CE

\begin{tabular}{|c|c|c|c|c|c|c|}
\hline Method & EI & $n=5$ & $n=10$ & $\mathrm{CE}$ & $n=5$ & $n=10$ \\
\hline 2nd $N M$ & $2^{\frac{1}{n+n^{2}}}$ & 0.0666 & 0.0181 & $2^{\frac{1}{3} n^{3}+2 n^{2}+\frac{2}{3} n}$ & 0.0210 & 0.0037 \\
\hline 4th $B C S T$ & $4 \frac{1}{n+2 n^{2}}$ & 0.0727 & 0.0190 & $4 \frac{1}{\frac{2}{3} n^{3}+5 n^{2}+\frac{1}{3} n}$ & 0.0190 & 0.0034 \\
\hline 5th $G G N$ & $5 \frac{1}{2 n+2 n^{2}}$ & 0.0833 & 0.0227 & $\begin{array}{c}5 \frac{1}{\frac{2}{3} n^{3}+5 n^{2}+\frac{4}{3} n} \\
1\end{array}$ & 0.0232 & 0.0042 \\
\hline 6th $C T V$ & $6 \frac{1}{3 n+2 n^{2}}$ & 0.0923 & 0.0260 & $6^{\frac{2}{3} n^{3}+6 n^{2}+\frac{7}{3} n}$ & 0.0244 & 0.0046 \\
\hline 4th $P M$ & $4 \frac{1}{n+2 n^{2}}$ & 0.0727 & 0.0190 & $\begin{array}{c}4 \frac{1}{3} n^{3}+4 n^{2}+\frac{2}{3} n \\
1\end{array}$ & 0.0275 & 0.0054 \\
\hline 5th $P M$ & $5 \frac{1}{2 n+2 n^{2}}$ & 0.0833 & 0.0227 & $\begin{array}{c}5^{\frac{1}{3} n^{3}+5 n^{2}+\frac{5}{3} n} \\
1\end{array}$ & 0.0285 & 0.0058 \\
\hline 6th $P M$ & $6^{\frac{1}{2 n+2 n^{2}}}$ & 0.1000 & 0.0272 & $6^{\frac{1}{3} n^{3}+5 n^{2}+\frac{5}{3} n}$ & 0.0342 & 0.0070 \\
\hline
\end{tabular}
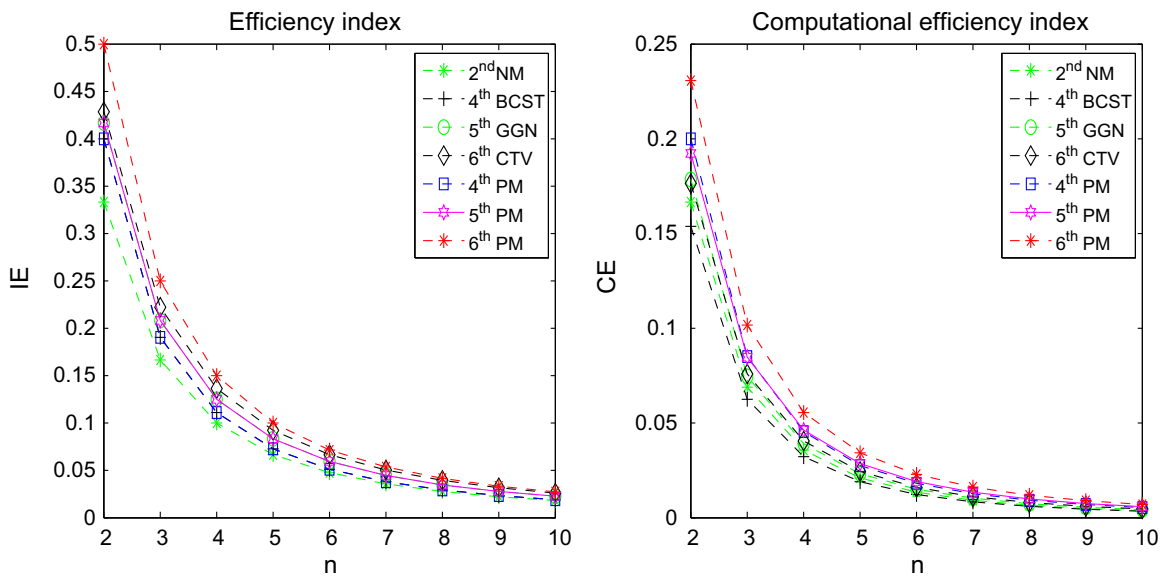

Fig. 1 Comparison of efficiency index and computational efficiency index

But, it must be as follows:

Table 1 Comparison of EI and CE

\begin{tabular}{|c|c|c|c|c|c|c|}
\hline Method & EI & $n=5$ & $n=10$ & $\mathrm{CE}$ & $n=5$ & $n=10$ \\
\hline 2nd $N M$ & $2^{\frac{1}{n+n^{2}}}$ & 1.0234 & 1.0063 & $2 \frac{1}{\frac{1}{3} n^{3}+2 n^{2}+\frac{2}{3} n}$ & 1.0073 & 1.0013 \\
\hline 4th $B C S T$ & $\frac{1}{4 n+2 n^{2}}$ & 1.0255 & 1.0066 & $\begin{array}{c}\frac{1}{\frac{2}{3} n^{3}+5 n^{2}+\frac{1}{3} n} \\
1\end{array}$ & 1.0066 & 1.0012 \\
\hline 5th $G G N$ & $5 \frac{1}{2 n+2 n^{2}}$ & 1.0272 & 1.0073 & $\begin{array}{c}5 \frac{2}{3} n^{3}+5 n^{2}+\frac{4}{3} n \\
1\end{array}$ & 1.0075 & 1.0014 \\
\hline 6th $C T V$ & $6 \frac{1}{3 n+2 n^{2}}$ & 1.0279 & 1.0078 & $6^{\frac{2}{3} n^{3}+6 n^{2}+\frac{7}{3} n}$ & 1.0073 & 1.0014 \\
\hline 4th $P M$ & $4 \frac{1}{n+2 n^{2}}$ & 1.0255 & 1.0066 & $\begin{array}{c}4 \frac{1}{3} n^{3}+4 n^{2}+\frac{2}{3} n \\
1\end{array}$ & 1.0096 & 1.0019 \\
\hline 5th $P M$ & $5 \frac{1}{2 n+2 n^{2}}$ & 1.0272 & 1.0073 & $\begin{array}{c}5^{\frac{1}{3} n^{3}+5 n^{2}+\frac{5}{3} n} \\
1\end{array}$ & 1.0092 & 1.0019 \\
\hline 6th $P M$ & $6^{\frac{1}{2 n+2 n^{2}}}$ & 1.0303 & 1.0082 & $6^{\frac{1}{3} n^{3}+5 n^{2}+\frac{5}{3} n}$ & 1.0103 & 1.0021 \\
\hline
\end{tabular}



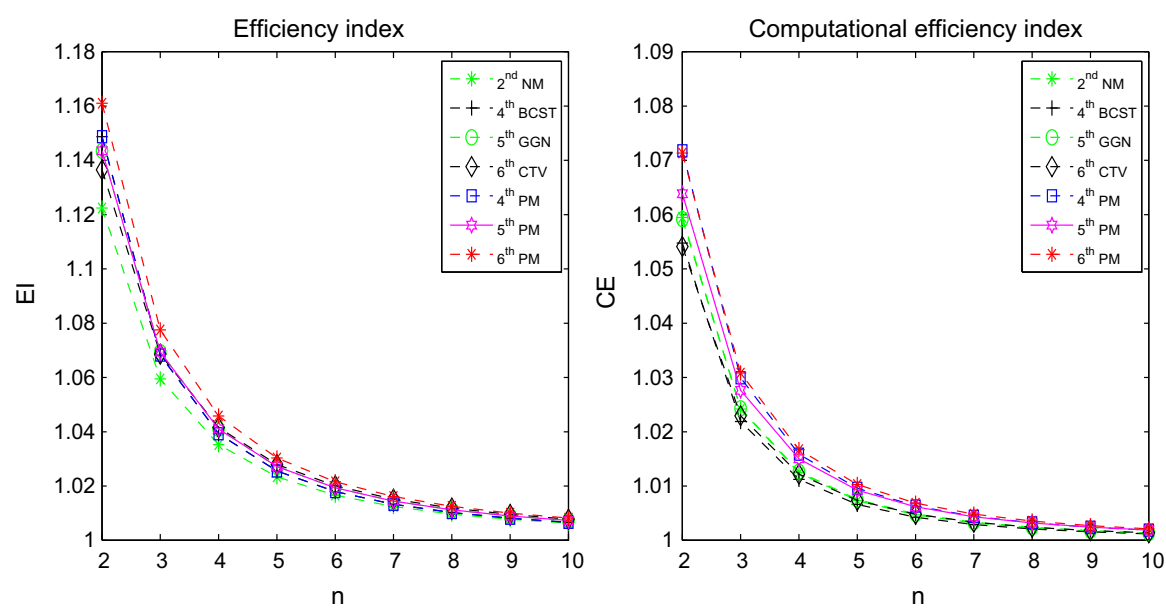

Fig. 1 Comparison of efficiency index and computational efficiency index 